\title{
A Short History of High-Voltage Direct Current Cables and Space Charge Measurements at Delft University of Technology
}

\author{
Peter Morshuis* \\ Solid Dielectric Solutions, Herensteeg 17, 2311 SG Leiden, The Netherlands
}

(Received December 12, 2016; accepted March 27, 2017)

Keywords: space charge, PEA, HVDC, electrical insulation

Around 1990, the attention for subsea high-voltage direct current (HVDC) cables strongly increased especially due to the deployment of a large number of HVDC connections in the northwest of Europe. In Delft, this triggered the first research projects in the field of HVDC insulation. At that time, other industries also approached the high-voltage laboratory of Delft to start accumulating more knowledge in this field. In the early 1990s, Professor Kreuger led the high-voltage laboratory and one of his mottos was "DC does not exist". This was used to motivate the students and make them aware of the fact that many different operating conditions are present under DC. The polarizations of the insulation and especially of the interfaces in the insulation are far from instantaneous, and the electric field in the insulation only gradually reaches a steady state. While a number of polarization and charging processes can be modelled, a more precise picture of the electric field distribution under different operating conditions is obtained by space charge measurements. This information is essential in the design process. For cable systems in operation, the proper knowledge of the electric and thermal stress distributions allows these systems to be operated more reliably and economically.

\section{Introduction}

In this paper, a chronological description of the developments in the field of high-voltage direct current (HVDC) insulation, which took place at Delft University of Technology's highvoltage laboratory, is given. It is shown how, initially, an in-depth treatment of the electric field distributions under HVDC led to increased insight into the electric stress in cable systems under different operating conditions. Gradually, the importance of space charge accumulation became clear, especially under voltage polarity reversal conditions. This led to the introduction of the pulsed electroacoustic (PEA) method developed by Professor Tatsuo Takada's group, which allowed nondestructive measurements to be made. ${ }^{(1)}$ Initially used on mass-impregnated paper cables and later on polymeric systems, the PEA method has led to an enormous improvement in the understanding of charge dynamics especially in polymeric systems. There is no doubt that the design and development of polymeric cables for HVDC received an incredible boost by the introduction of nondestructive space charge measurement techniques such as PEA.

*Corresponding author: e-mail: peter.morshuis@dielectrics.nl http://dx.doi.org/10.18494/SAM.2017.1557 
At the start of the 21st century, a European project started on HVDC cable systems, and in Delft, the work focused more on the understanding of the space charge dynamics in cable accessories. In particular, for HVDC applications, the cable accessories pose the biggest challenges to the designer. In fact, when we are discussing HVDC insulation systems, it is all about interfaces. Probably, it is clear that the macroscopic interfaces in high-voltage cable joints need to be carefully designed. More recently, we have seen the focus shift to mesoscopic and nanoscopic interfaces because of their potential benefit in terms of breakdown voltage and space charge mitigation.

In this paper, we will focus on how space charge measurements and, in particular, PEA measurements helped in understanding the complicated charging processes in HVDC cables and accessories. Some thoughts on future work to be done in the field of HVDC insulation with an emphasis on high-voltage cables and space charge will conclude the paper.

\section{Determining the Electric Field Distribution}

In the first part of this section, the different cable operating conditions are described, and in the second part, how to calculate the electric field distributions under these conditions is shown.

\subsection{Operating conditions}

HVDC cable systems are designed for electric fields under steady-state conditions and also for transient conditions. Whereas for AC cables, transient conditions would refer only to lightning and switching transients, for HVDC systems, transients also occur due to voltage polarity reversals and changing load currents. In fact, field calculations are far more complex at DC voltages than at $\mathrm{AC}$ voltages. This is largely due to the nonlinear behavior of the insulation as well as to the polarization and charging time constants involved. Perhaps most importantly, temperature plays a very important role in determining the electric field distribution. Any change in temperature will result in a change in electric field distribution.

Kreuger $^{(2)}$ argued that there are different stages for which the electric field can be characterized. In Fig. 1, a graphical representation of these stages is given.

(a)

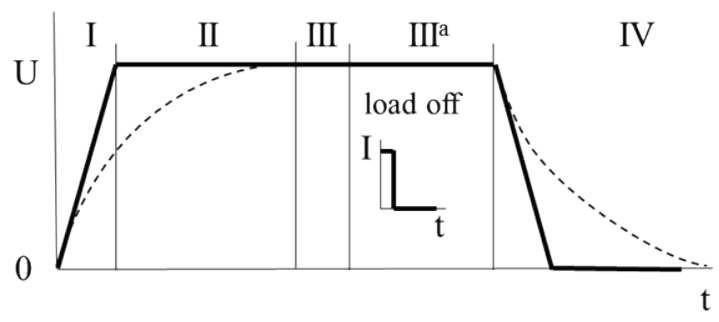

(b)

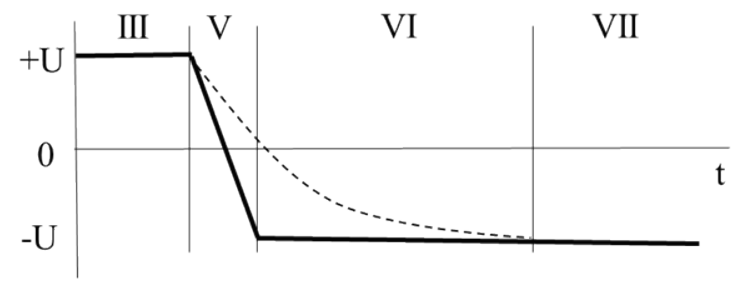

Fig. 1. Different stages (a) when switching on and off a DC voltage and (b) after a polarity reversal. The broken lines represent the growth and decay of internal polarization and space charge. After Kreuger. ${ }^{(2)}$ 
In stage I, the voltage is applied to the cable. Initially, the cable is not polarized and contains no space charge. There is no temperature drop across the insulation yet. The electric field in the cable and its accessories is determined by the geometry and permittivity only. We speak of a capacitively distributed electric field. During stage II, even though the applied voltage is now constant, the electric field is changing from a capacitively to a resistively distributed field. In this stage, polarization processes take place and space charge is accumulating. Finally, in stage III, the electric field is resistively distributed. In stages I-III, a load current may be present, resulting in the gradual buildup of a temperature drop across the cable insulation. This temperature drop will affect the duration of these stages. In case the load current is switched off, we have to introduce stage III ${ }^{\mathrm{a}}$, which is a special case of stage III. In stage IV, the applied voltage is lowered to zero but the electric field is still present due to the polarization and space charge in the insulation. In the case of a voltage polarity reversal, stage $\mathrm{V}$ is defined in which the applied voltage changes polarity. After the voltage polarity reversal, the internal charges will be redistributed during stage VI until, finally, in stage VII, the electric field becomes stable.

\subsection{Determining the electric field at different stages}

The distribution of the electric field at DC voltage is determined by the electrical conductivity and permittivity of the insulation, the geometry, the applied voltage, and the space charge density distribution. Because the conductivity is a complex function of temperature and local electric field, the determination of the electric field distribution is not straightforward.

Usually, an Arrhenius-type relationship is found for the temperature dependence of the conductivity:

$$
\sigma(T)=A \exp \left(-\frac{E_{a}}{k_{B} T}\right)
$$

where $\sigma$ is the electrical conductivity $(\mathrm{S} / \mathrm{m}), T$ the temperature $(\mathrm{K}), A$ a constant $(\mathrm{S} / \mathrm{m}), E_{a}$ the activation energy $(\mathrm{J})$, and $k_{B}$ Boltzmann's constant $(\mathrm{J} / \mathrm{K})$.

The dependence of the conductivity on electric field is much more complex and is in fact determined by the space charge dynamics of the insulation. This can to a certain extent be modelled, and in Sects. 4 and 5, this will be discussed in more detail. For now, we will take a simple exponential relationship between the conductivity and the electric field:

$$
\sigma(T, E)=A \exp \left(-\frac{E_{a}}{k_{B} T}\right) \exp (\gamma E)
$$

where $\gamma$ is the field-dependent coefficient $(\mathrm{m} / \mathrm{V})$.

The general effect of the temperature dependence is an inversion of the electric field distribution as shown in Fig. 2. In the absence of a load current, the highest electric field is found near the conductor and the lowest field is found near the ground sheath, similarly to an AC cable. As soon as a load current is switched on, a temperature drop is created across the insulation. Owing to the temperature dependence of the conductivity, the highest field is now "pushed" towards the ground sheath.

Having modeled the conductivity as a function of temperature and electric field, we can now calculate the electric field distribution for all stages mentioned. 


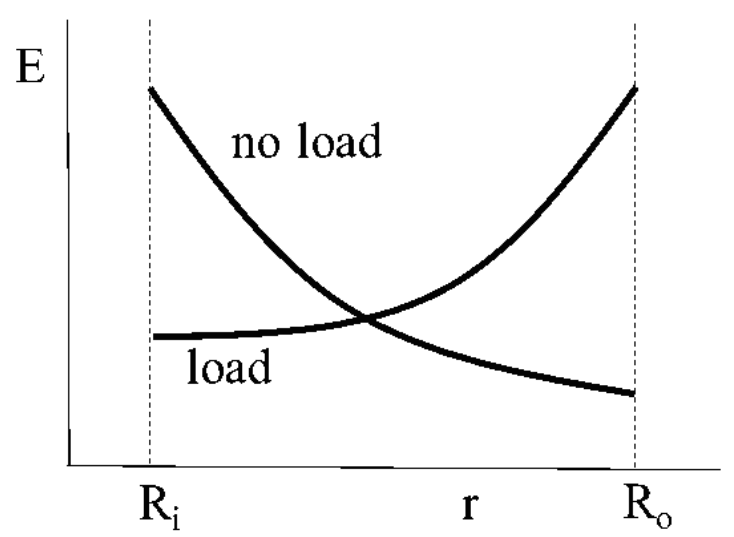

Fig. 2. Stable DC fields under load and no-load conditions. Here, no dependence of the conductivity on the electric field is assumed.

Stage I - The electric field is capacitively distributed and can be calculated resorting to the applied voltage and geometry, and, in the case of accessories, to the permittivity.

Stage II - The electric field cannot be calculated using an expression in closed mathematical form and has to be calculated numerically, taking into account the temperature and field dependences of the conductivity.

Stage III - The electric field distribution including the effects of temperature and field dependences is given by

$$
E(r)=U \frac{r^{k-1} \exp (-\gamma E)}{\int_{R_{i}}^{R_{o}} r^{k-1} \exp (-\gamma E) d r},
$$

where $=\frac{\alpha \Delta T}{\ln \left(\frac{R_{o}}{R_{i}}\right)}$. This can be calculated numerically only.

If we neglect the field dependence for simplicity, we can derive a closed form equation for the electric field distribution for a given temperature drop across the insulation: ${ }^{(3)}$

$$
E(r)=U \frac{k}{R_{o}\left[1-\left(\frac{R_{i}}{R_{o}}\right)^{k}\right]}\left(\frac{r}{R_{o}}\right)^{k-1} .
$$

Note that, for $k=1$, the electric field does not depend on the radius, and thus the electric field is uniform. For values of $k$ larger than 1, the electric field distribution is inversed.

\section{Modeling the Space Charge Profiles in Cables}

If we have full access to the temperature and field dependences of the electrical conductivity, we can numerically calculate the distribution of the electric field for all stages.

We start with the initial distributions of the electric field $E(r)$, the temperature $T(r)$, the current density $J(r)$, the space charge density $\rho(r)$, and the conductivity $\sigma(r)$. 
A first approximation of the temperature distribution (disregarding the leakage current losses) is given by

$$
T(r)=T\left(R_{o}\right)+\frac{W_{c}}{2 \pi \lambda_{T}} \ln \left(\frac{R_{o}}{r}\right)
$$

where $W_{c}$ is the conductor loss per meter $(\mathrm{W} / \mathrm{m})$ and $\lambda_{T}$ is the thermal conductivity of the insulation (W/ $\mathrm{m} / \mathrm{K}$ ). In case the leakage current losses need to be accounted for, the calculation becomes more complex.

Then, the flowchart as shown in Fig. 3 is followed.

\section{Measurement of Space Charge Profiles in Cables}

The development of space charge measurement systems, such as the pulsed electroacoustic method, made it possible for the first time to compare the calculated electric field distribution with the real field distribution. In Fig. 4, a comparison of calculated and measured space charge distributions in cross-linked polyethylene (XLPE) cable of $4.5 \mathrm{~mm}$ insulation thickness ${ }^{(4)}$ is shown.

It is clearly seen that, at $t=0$, the cable is space-charge-free and the resulting electric field distribution is as expected, i.e., a capacitively distributed field. In the course of time, the positive space charge moves in the direction of the ground sheath owing to the temperature dependence of the conductivity. The result is an inversion of the electric field. It can be concluded that the results of the calculations approximate reasonably well the measured values of the space charge density and thus the electric field. The main reason for the discrepancy is the fact that the applied voltage was sufficiently high to enable charge trapping in the insulation. This phenomenon is not

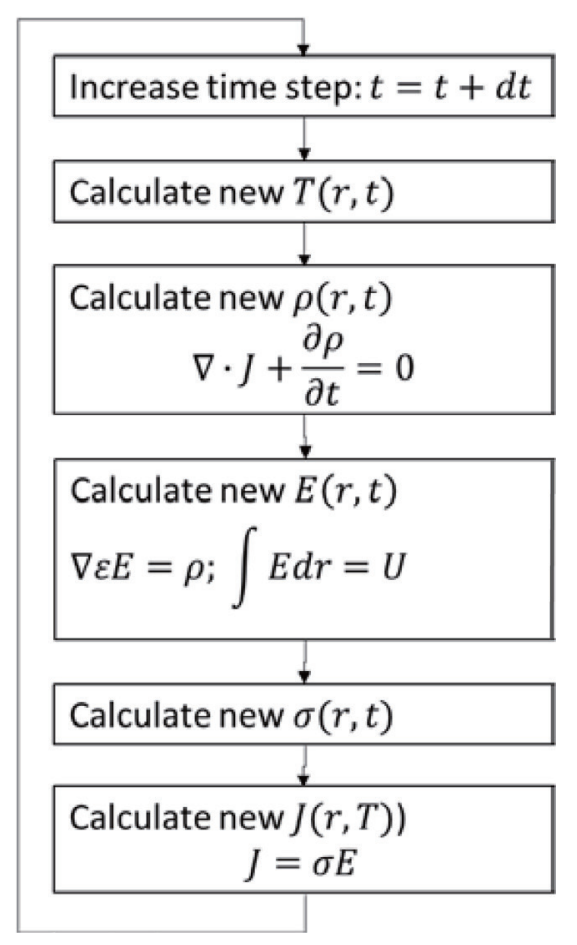

Fig. 3. Flowchart of numerical calculation of the electric field distribution. ${ }^{(3)}$ 


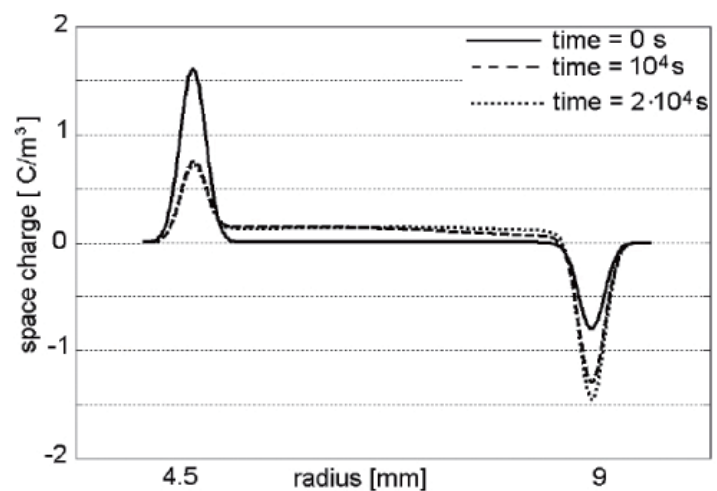

(a)

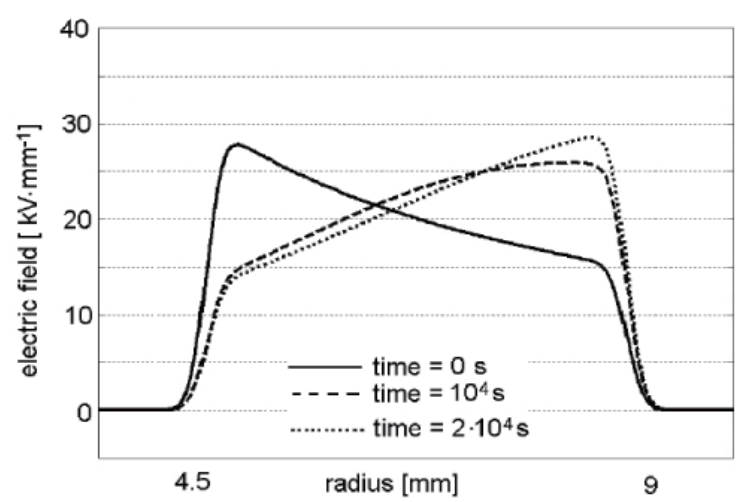

(c)

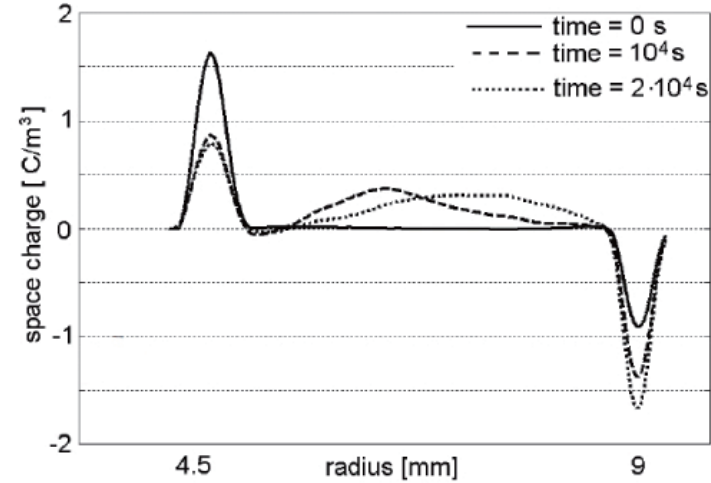

(b)

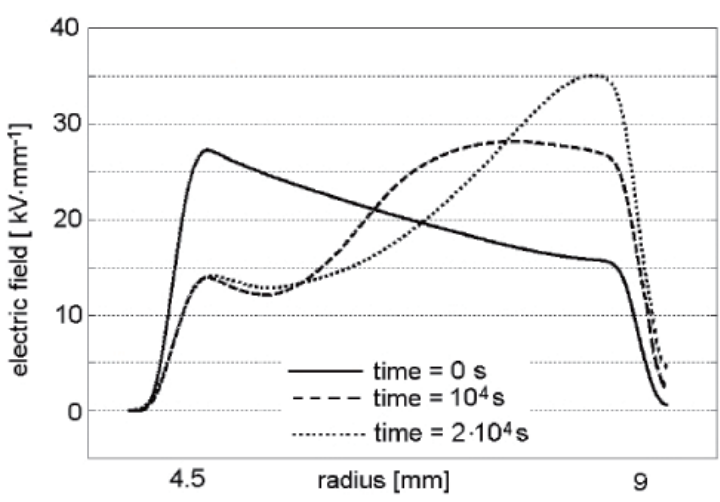

(d)

Fig. 4. (a) Calculated and (b) measured space charge distributions in an XLPE cable with an initial temperature drop across the insulation of $20{ }^{\circ} \mathrm{C}$, applied voltage: $90 \mathrm{kV}$; (c) calculated and (d) measured (obtained from measured space charge profile) electric field distributions. Measurements were taken at $t=0,3$, and $6 \mathrm{~h}$.

accounted for in the model used for the calculations. Furthermore, it is clear that a considerable amount of time is required for the space charge accumulation to reach a steady-state level. This is an important fact to be taken into account in testing full-size HVDC cables for space charge. Indeed, in a new IEEE standard to appear in 2017, this is described in detail. ${ }^{(5)}$

\section{Modeling and Measuring of Space Charge Profiles in Cable Accessories}

A next step in the use of the PEA method was its application to HVDC cable accessories. ${ }^{(4)}$ In a first approach, an ethylene propylene rubber-insulated (EPR) cable joint on an XLPE cable was modeled using a dual-dielectric cable, as shown in Fig. 5. In Fig. 6, a comparison is shown between the calculated and measured space charge distributions. In this particular case, the applied voltage was chosen to be relatively low $(30 \mathrm{kV})$ to minimize any trapped space charge accumulation. An initial stable temperature drop of $22{ }^{\circ} \mathrm{C}$ was present. Now, it is seen that the calculated and measured values match quite well. Indeed, the measured electric field distribution resembles quite well the measured distribution. Interestingly, at time $t=0$, already some signal is observed at the location of the interface between XLPE and EPR owing to the discontinuity of the permittivity. 


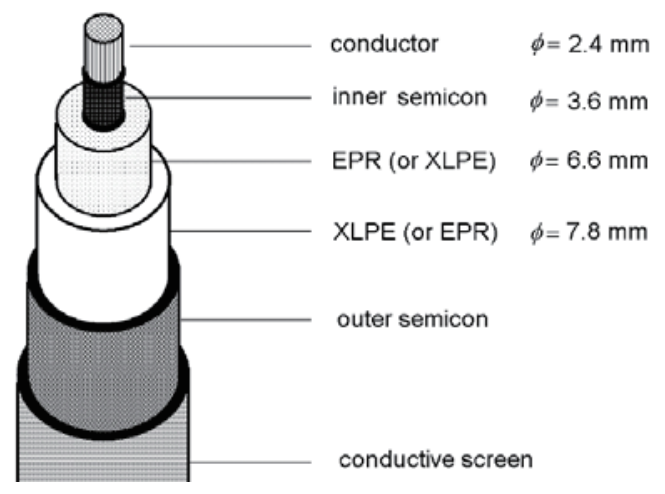

Fig. 5. Schematic representation of a dual-dielectric cable that was used to model a cable joint.

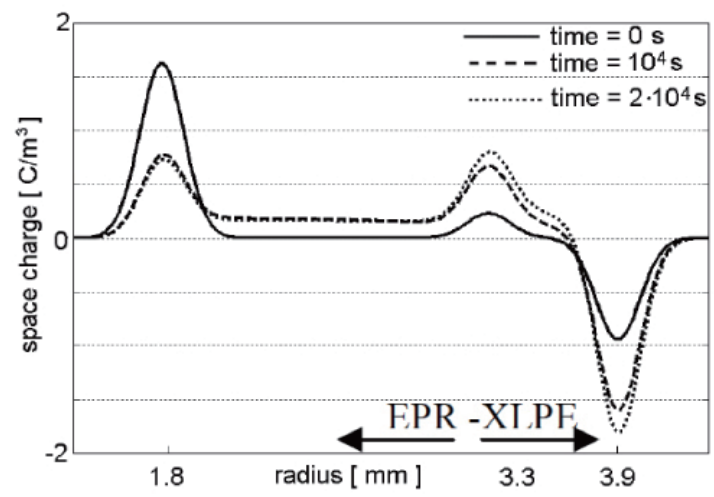

(a)

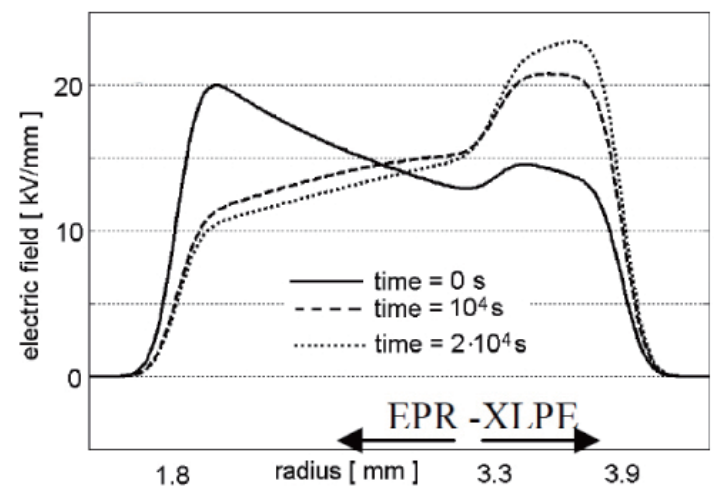

(c)

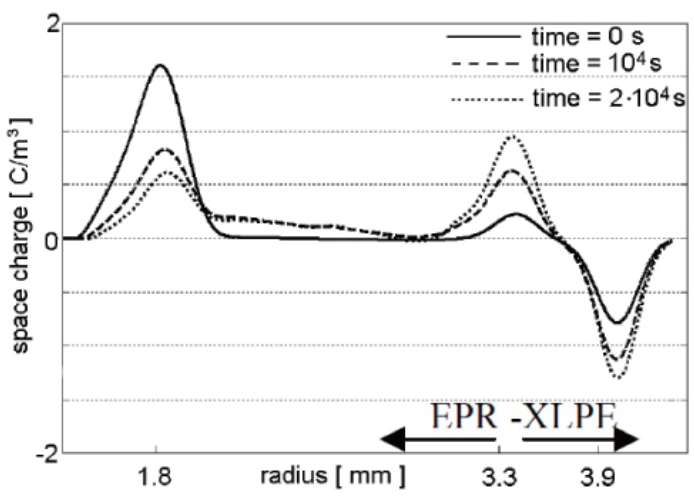

(b)

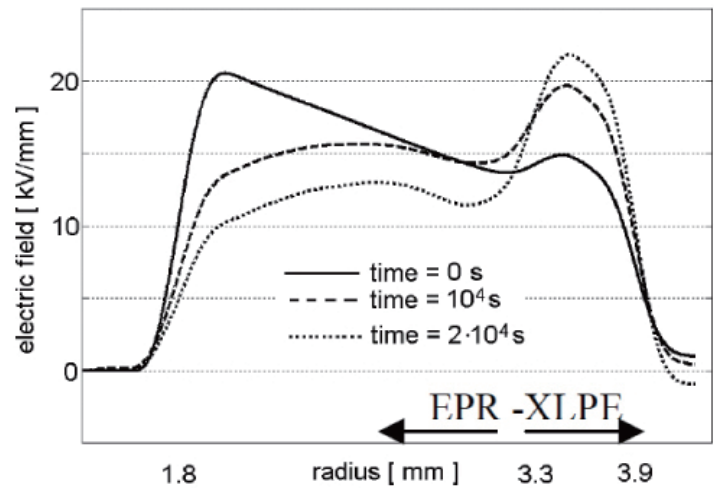

(d)

Fig. 6. (a) Calculated and (b) measured space charge distributions in a dual dielectric EPR/XLPE cable with an initial temperature drop across the insulation of $22{ }^{\circ} \mathrm{C}$ and applied voltageof $30 \mathrm{kV}$. (c) Calculated and (d) measured (obtained from measured space charge profile) electric field distributions. Measurements were taken at $t=0,3$, and $6 \mathrm{~h}$.

This should not be mistaken for space charge. In the course of time, we see an increase in positive space charge density at this interface, which can be modelled by assuming interfacial polarization according to the Maxwell-Sillars-Wagner model. The result is a further increase in electric field in the XLPE layer. 
A further step ${ }^{(4)}$ in using the PEA method on cable accessories was made when a mediumvoltage-size cable joint was built as shown in Fig. 7. The total insulation thickness of the joint was $4 \mathrm{~mm}$. In Fig. 8, a comparison is shown between the calculated and measured space charge profiles. In this case, the applied voltage was rather high $(80 \mathrm{kV})$ and trapped space charge accumulation was expected. This becomes clear when the calculated and measured space charge profiles are compared. The charge accumulation at the interface between EPR and XLPE is quite well calculated, but the positive charge moving into the XLPE from the high-voltage electrode is not accounted for by the model calculations. The result is that the amount of field enhancement in the EPR layer is underestimated by the model.

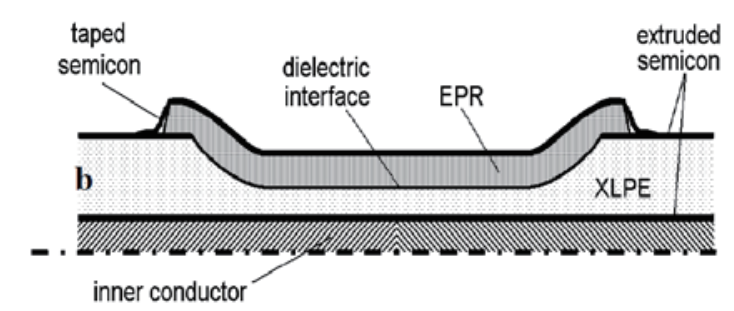

Fig. 7. Schematic representation of a medium-voltage-size cable joint.

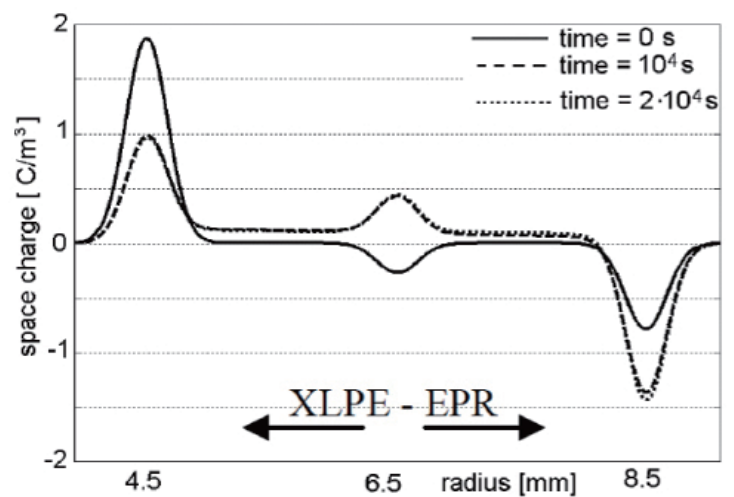

(a)

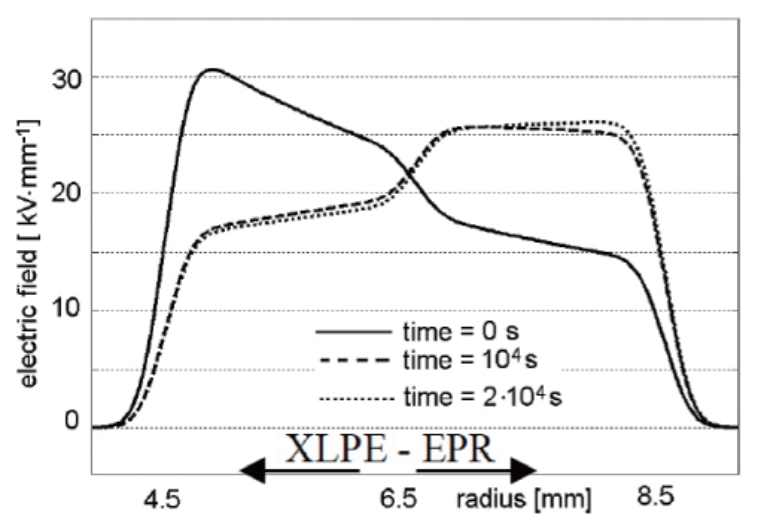

(c)

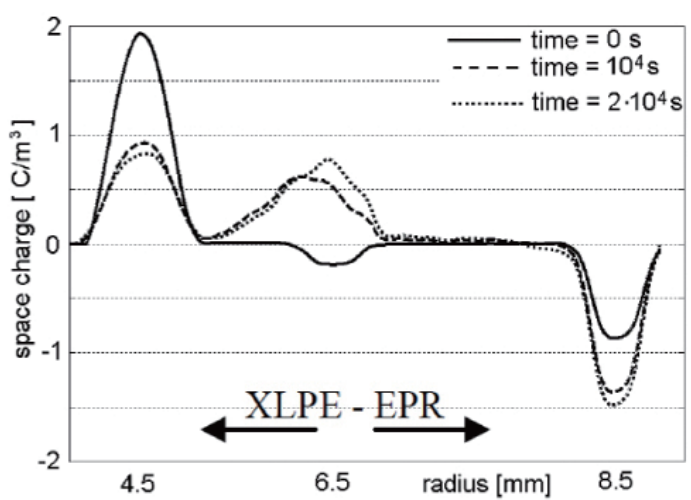

(b)

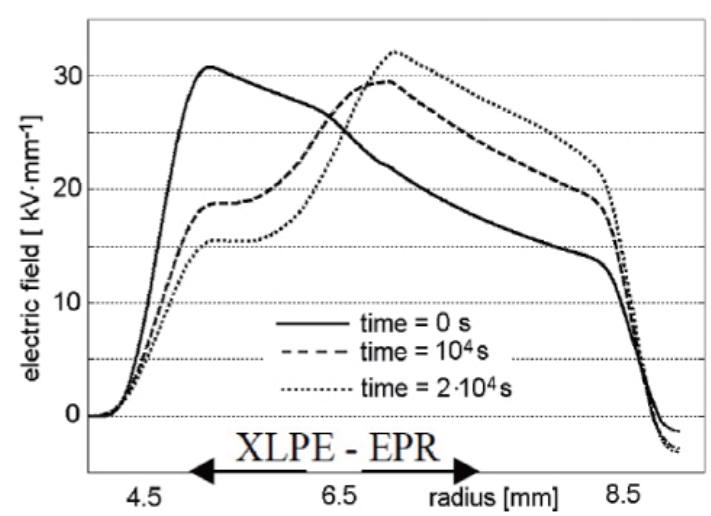

(d)

Fig. 8. (a) Calculated and (b) measured space charge distributions in a medium-voltage-size EPR/XLPE cable with an initial temperature drop across the insulation of $20^{\circ} \mathrm{C}$ and applied voltage of $80 \mathrm{kV}$. (c) Calculated and (d) measured (obtained from measured space charge profile) electric field distributions. Measurements were taken at $t=0$, 3 , and $6 \mathrm{~h}$. 
An in-depth treatment of the results of the European HVDC project with an emphasis on space charge at interfaces in cable systems can be found in a series of three papers. ${ }^{(6-8)}$ In conclusion, the added advantage of space charge measurements next to model calculations is clear: in space charge measurements, the trapped space charge is also visible.

\section{Space Charge Tests in Full-Size Cables}

Recently, the number of activities in space charge measurements on full-size cable systems has increased. In a joint project, ${ }^{(9)}$ the universities of Montpellier and Leicester started a long-term space charge monitoring project on a $200 \mathrm{kV}$ full-size cable during a voltage source converters (VSC) ageing programme according to Cigré TB496 together with cable manufacturers.

Furthermore, an IEEE Dielectrics and Electrical Insulation Society (DEIS) Technical Committee devised a series of guidelines for performing space charge measurements on full-size cable systems during prequalification tests and type tests. ${ }^{(10)}$ These recommendations were then further developed and will appear in an IEEE standard ${ }^{(5)}$ in 2017.

\section{Potential Future Work}

The developments in introducing HVDC cable systems are fast. When we focus on the challenges of industry, we can only conclude that they are enormous. Perhaps one single example is sufficient to underline this. In Germany, it has been decided quite recently that all extensions and reinforcements of the existing grid should be underground. In practice, this means that, in a relatively short period of time, some thousands of kilometers of HVDC cables have to be installed. There is no project in the world that comes close to this. In particular, if one realizes that for land cable, the number of joints to be installed on-site is huge, and the challenges become clear. One potential application of space charge measurements is the on-line use of PEA on land-based HVDC cable accessories. Using PEA on-line would not only give insight into the real stress distribution of the accessories and hence give guidelines for safe use, but it would also allow optimal use of the cable system, both thermal and electrical. The transmission system operators could potentially benefit significantly.

\section{Conclusions}

Since the early 1990s, the introduction of nondestructive space charge measurement methods such as the PEA method has helped tremendously in developing polymeric HVDC cables and accessories. The complex field distributions that occur in HVDC cable systems can only be fully studied and understood by combining a modeling approach with the recording of real data.

Whereas the PEA method initially was used on laboratory specimens only, by now, the method is used on full-size cable systems. In 2017, the first (IEEE) standard on space charge measurements on polymeric cable systems up to $550 \mathrm{kV}$ will appear. It is only a matter of time before space charge measurements will become standard during prequalification and type tests.

Professor Tatsuo Takada and his team have proved to be very important in developing the pulsed electroacoustic method. As such, they have indirectly contributed to the large-scale introduction of HVDC polymeric cable systems. 


\section{Acknowledgments}

The work described in this paper was mainly performed by my Ph.D. students, Marc Jeroense and Riccardo Bodega, supported by a number of MSc students. Marc Jeroense went to Professor Takada's laboratory in the early 1990s to learn how to build a PEA system. I am still grateful to Professor Takada for offering his indispensable help and allowing Marc Jeroense to stay in his lab, build a PEA system, and bring the PEA system to Delft. Thank you.

\section{References}

1 T. Takada, T. Maeno, and H. Kushibe: Proc. 1985 5th Int. Symp. Electrets (IEEE, 1985). doi: 10.1109/ISE.1985.7341521

2 F. H. Kreuger: Industrial High DC Voltage (Delft University Press, Delft, 1995).

3 M. J. P. Jeroense: Charges and Discharges in HVDC Cables (Ph.D. Thesis Delft University of Technology, Delft University Press, Delft, 1997).

4 R. Bodega: Space Charge Accumulation in Polymeric High Voltage DC Cable Systems (Ph.D. Thesis Delft University of Technology, Delft, 2006).

5 G. Mazzanti, G. Chen, J. C. Fothergill, N. Hozumi, J. Li, M. Marzinotto, F. Mauseth, P. Morshuis, C. Reed, A. Tzimas, and Kai Wu: IEEE Standard P1732 (2017).

6 D. Fabiani, G. C. Montanari, C. Laurent, G. Teyssedre, P. H. F. Morshuis, R. Bodega, L. A. Dissado, A. Campus, and U. H. Nilsson: IEEE Electr. Insul. Mag. 23(6) (2007) 11.

7 S. Delpino, D. Fabiani, G. C. Montanari, C. Laurent, G. Teyssedre, P. H. F. Morshuis, R. Bodega, and L. A. Dissado: IEEE Electr. Insul. Mag. 24(1) (2008) 14.

8 D. Fabiani, G. C. Montanari, C. Laurent, G. Teyssedre, P. H. F. Morshuis, R. Bodega, and L. A. Dissado: IEEE Electr. Insul. Mag. 24(2) (2008) 5.

9 A. Tzimas, L. Boyer, P. Mirebeau, S. Dodd, J. Castellon, and P. Nothingher: Proc. 2016 IEEE Conf. Electrical Insulation and Dielectric Phenomena (IEEE, 2016). doi: 10.1109/ICD.2016.7547540

10 G. Mazzanti, G. Chen, J. C. Fothergill, N. Hozumi, J. Li, M. Marzinotto, F. Mauseth, P. Morshuis, C. Reed, A. Tzimas, and Kai Wu: IEEE Trans. Dielectr. Electr. Insul. 22 (2015) 21. 